\title{
Outcomes of Transurethral Resection of Prostate in Patients with and without Renal Failure Secondary to an Enlarged Prostate
}

\author{
Mudassir Hussain, Abdul Khalique, Pardeep Kumar Maheshwari, Usman Qamar and Asad Shehzad \\ Department of Urology, Sindh Institute of Urology and Transplantation, Karachi, Pakistan
}

\begin{abstract}
The natural history of benign enlargement of the prostate is variable and ranges from mild symptoms to chronic retention and renal failure. In this study, the outcomes of patients with urinary retention alone were compared with those of chronic retention and renal failure caused by an enlarged prostate. The first group had 79, while the second group had 20 patients included. The mean maximum flow rate after transurethral resection of the prostate (TURP) in the two groups was $16.9 \pm 7.9$ vs. $14.6 \pm 4.1$ $\mathrm{ml} / \mathrm{sec}$ ( $\mathrm{p}$ value $>0.05$ ), and the mean post-void volume was $15.1 \pm 27.6 \mathrm{vs} .21 .7 \pm 35.7 \mathrm{ml}$ ( $\mathrm{p}$ value $>0.05$ ), respectively. However, the residual symptoms after surgery were higher in the chronic retention group. It was concluded that patients, with chronic retention experience and higher postoperative residual storage symptoms, after transurethral resection of the prostate, are able to void without a catheter and their renal functions were stabilised.
\end{abstract}

Key Words: Transurethral resection of the prostate, Prostatic hyperplasia, Renal insufficiency, Urinary bladder neck obstruction.

How to cite this article: Hussain M, Khalique A, Maheshwari PK, Qamar U, Shehzad A. Outcomes of Transurethral Resection of Prostate in Patients with and without Renal Failure Secondary to an Enlarged Prostate. J Coll Physicians Surg Pak 2021; 31(10):1247-1249.

The natural history of benign prostatic hyperplasia (BPH) progression and its consequences vary from mild symptoms to chronic retention and loss of renal function. Most patients (79.5\%) are asymptomatic, while few $(2-5 \%)$ progress to urinary retention. ${ }^{1}$ Among these, some develop chronic urinary obstruction, bladder dysfunction, upper tract dilatation, and subsequent renal failure.

There is a lack of reliable data on the management and outcomes of those with chronic urinary retention and renal failure. The pathophysiology is related to high-pressure storage and voiding, leading to detrusor muscle hypertrophy, and reduces compliance that results in renal dysfunction. ${ }^{2}$ Even after relieving outlet obstruction through transurethral resection of the prostate, the bladder dysfunction continues to cause symptoms and risk deterioration of the upper tract.

The knowledge of outcomes of TURP in patients with chronic urinary retention is relevant for counselling and consent. These patients expect catheter-free voiding and stable renal functions that might be affected by the degree of bladder dysfunction, and are difficult to predict.

Correspondence to: Dr. Mudassir Hussain, Department of Urology, Sindh Institute of Urology and Transplantation, Karachi, Pakistan

E-mail: mudassir.hussain120@gmail.com

Received: January 19, 2021; Revised: June 16, 2021;

Accepted: June 23, 2021

DOI: https://doi.org/10.29271/jcpsp.2021.10.1247
They also need to be closely followed to monitor their renal functions and upper tract hydronephrosis in the postoperative period.

This study was aimed to compare the early outcomes of patients with acute and chronic urinary retention after transurethral resection of the prostate.

The study was conducted at the Department of Urology, Sindh Institute of Urology and Transplantation from August 2019 to August 2020 after the approval from Ethical Review Committee. There were two study groups, the first had acute retention of urine with normal renal function, and the second had chronic retention of urine with renal dysfunction. Patients with diabetes, hypertension and prostate cancer were excluded.

The data were analysed using the software IBM SPSS version 20.0. The quantitative variables, such as age, PSA levels, prostate volume, maximum flow rate, post-void volume, and serum creatinine levels, were expressed as mean $\pm S D$; and were compared using the student t-test. The mean preoperative and postoperative serum creatinine levels for each group were also compared using paired sample t-test. The qualitative variables were expressed as proportions, and compared using the Chisquare test with a $p$ value of less than 0.05 considered significant.

There were 99 patients included in the study. Among them, 79 were with urinary retention alone, while 20 had chronic urinary retention. The mean age was $62.9 \pm 7.7$ years, prostate-specific antigen $4.5 \pm 4.5 \mathrm{ng} / \mathrm{ml}$, and the prostate size $46.2 \pm 23.8 \mathrm{ml}$. 
These parameters were compared and no statistical difference was found (Tablel).

After the surgery, the percentage of residual lower urinary tract symptoms was slightly higher in the chronic urinary retention group; however, it did not reach statistical significance. The maximum flow rate, average flow rate, and post-void volume were compared and no statistical difference was found among them (Tablel).

Table I: Descriptive statistics and the comparison of postoperative outcomes.

\begin{tabular}{|l|c|c|c|}
\hline & $\begin{array}{c}\text { Urinary retention } \\
\mathbf{n}=\mathbf{7 9} \\
\text { mean (SD) }\end{array}$ & $\begin{array}{c}\text { Chronic urinary } \\
\text { retention, } \\
\mathbf{n}=\mathbf{2 0} \text { mean (SD) }\end{array}$ & p-value \\
\hline Age (years) & $63.0(8.2)$ & $62.3(5.5)$ & 0.717 \\
\hline PSA (ng/ml) & $4.16(4.7)$ & $5.79(3.4)$ & 0.182 \\
\hline Prostate size (ml) & $47.24(24.1)$ & $41.95(22.5)$ & 0.378 \\
\hline Qmax (ml/sec) & $16.9(7.9)$ & $14.6(4.1)$ & 0.289 \\
\hline Qavg (ml/sec) & $8.3(4.3)$ & $7.1(2.2)$ & 0.151 \\
\hline Post void volume (ml) & $15.1(27.6)$ & $21.7(35.7)$ & 0.374 \\
\hline Nocturia & $45(57 \%)$ & $12(60 \%)$ & 0.924 \\
\hline Frequency & $30(38 \%)$ & $8(40 \%)$ & 0.834 \\
\hline Urgency & $33(41.8 \%)$ & $10(50 \%)$ & 0.471 \\
\hline Urge incontinence & $20(25.3 \%)$ & $8(40 \%)$ & 0.421 \\
\hline Hesitancy & $19(24.0 \%)$ & $5(25 \%)$ & 0.921 \\
\hline Intermittency & $6(7.6 \%)$ & $4(20 \%)$ & 0.116 \\
\hline Good Stream & $62(78.5 \%)$ & $14(70 \%)$ & 0.458 \\
\hline Post void dribbling & $9(11.4 \%)$ & $3(15 \%)$ & 0.850 \\
\hline
\end{tabular}

The mean serum creatinine for the patients with urinary retention alone was $1.21 \pm 0.17 \mathrm{mg} / \mathrm{dl}$ preoperatively, and $1.25 \pm$ $0.18 \mathrm{mg} / \mathrm{dl}$ postoperatively. In the chronic retention group, the creatinine levels before surgery, i.e. preoperative, was $2.94 \pm$ $0.75 \mathrm{mg} / \mathrm{dl}$ (best creatinine after four to six weeks of bladder drainage); while, after the surgery the mean level of creatinine was $2.93 \pm 1.12 \mathrm{mg} / \mathrm{dl}$. There was no statistically significant difference in creatinine of chronic urinary retention group before and after the surgery $(p=0.947)$. However, a small difference was seen in the urinary retention group alone $(p=0.035)$.

Renal insufficiency coexists with prostatic enlargement in 5.9 to $11 \%$ of the elderly. However, its relation with bladder outlet obstruction is not defined. ${ }^{3}$ Those with hydroureteronephrosis and significant post-void volume were considered to have bladder outlet obstruction; and it is confirmed clinically, when they improve after bladder drainage. Considering age, the most common cause of such obstruction is an enlarged prostate, while others include urethral stricture and neurogenic lower tract dysfunction.

Risk factors for chronic urinary retention include high IPSS score, lower urinary flow, and high post-void volume. The obstructive nephropathy in these patients is the consequence of bladder dysfunction and high intravesical pressures. It has been shown that men with decreased bladder compliance are more likely to develop nephropathy $78 \%$, compared to men with normal bladder compliance $36 \%{ }^{4}$

In this study, the authors found that residual lower urinary tract symptoms, after prostate surgery, were common at early followup. In those with chronic retention, they were higher although not statistically significant (Table 1 ). The presence of greater bladder dysfunction explains the higher residual symptoms in the renal dysfunction group.

The overall mean maximum flow rate in our patients, after the surgery, was $16.4 \pm 7.3 \mathrm{ml} / \mathrm{sec}$, with no statistically significant difference between groups, i.e. $16.9 \pm 7.9$ vs. $14.6 \pm 4.1 \mathrm{ml} / \mathrm{sec}$ ( $p$ $=0.289$ ). Considering all of them were catheterised pre-operatively, this is a significant improvement.

TURP relieves the outlet obstruction, but it does not resolve bladder dysfunction. The impaired dynamics of the bladder are largely responsible for residual storage symptoms that have been reported to improve over time, spontaneously in most patients. However, a longer follow-up of these patients can help understand their natural history.

Since the majority of postoperative symptoms are the consequence of bladder dysfunction, they comprise of storage type, i.e. nocturia, frequency, and urgency.

In this study, higher storage symptoms were found in patients with renal failure. In contrast, the voiding symptoms were better in the latter group. This illustrates the critical role of bladder dysfunction in final outcomes of chronic retention.

The management of these symptoms is still debated. An RCT compared tamsulosin alone with tamsulosin and solifenacin after surgery for four weeks. They reported no statistical difference when compared to placebo. ${ }^{5}$ However, Sipal et al. have shown that solifenacin in the early postoperative period improved such symptoms. ${ }^{6}$ At the study department, these symptoms are managed conservatively; and anticholinergics are prescribed, when they are refractory.

The mean serum creatinine before prostate surgery was $1.21 \pm$ 0.17 in those with urinary retention and $2.94 \pm 0.75 \mathrm{mg} / \mathrm{dl}$ in those with chronic urinary retention. Renal functions remained stable after surgery at 4 weeks, i.e. $1.25 \pm 0.18$ and $2.93 \pm 1.12$ $\mathrm{mg} / \mathrm{dl}$, respectively. This is an important outcome for patients with chronic retention, who can void without a catheter with a little risk of renal function deterioration. This also demonstrates that relieving obstruction improves bladder dynamics and prevents further renal dysfunction in the short term.

The above findings are relevant for patients with chronic urinary retention. These patients should be informed that they will 
likely face residual storage symptoms and their voiding will not beas good as expected.

This study is limited by short follow-up, lack of urodynamic diagnosis and the use of direct questions about lower urinary tract symptoms instead of validated scale, such as the international prostate symptomsscore.

Transurethral resection of the prostate allows successful urethral voiding without deterioration of renal functions in elderly patients with chronic urinary retention. However, the residual symptoms are higher as compared to patients with urinary retention alone. These patients should be counselled about the need for close observation and postoperative medical treatment to control their symptoms.

\section{CONFLICT OF INTEREST:}

The authors declared no conflict of interest.

\section{AUTHORS' CONTRIBUTION:}

$\mathrm{MH}$ : Conception of design, data collection, analysis and interpretation of data.

$A K$ : Conception of idea, analysis and interpretation of data.

PKM, UQ: Analysis and interpretation of data.

AS: Critical review of the work and final approval.

\section{REFERENCES}

1. Fitzpatrick JM. The natural history of benign prostatic hyperplasia. BJU Int 2006; 97 (Suppl 2):3-6; discussion
21-22. doi: 10.1111/j.1464-410X.2006.06097.x.

2. Sinha S, Matai L. Is isolated bladder outlet obstruction associated with hydronephrosis? A database analysis. Neurourol Urodyn 2020; 39(8):2361-7. doi: 10.1002/nau. 24495.

3. Gerber GS, Goldfischer ER, Karrison TG, Bales GT. Serum creatinine measurements in men with lower urinary tract symptoms secondary to benign prostatic hyperplasia. Urol 1997; 49(5):697-702. doi: 10.1016/S0090-4295(97)00 069-1.

4. Comiter CV, Sullivan MP, Schacterle RS, Cohen LH, Valla SV. Urodynamic risk factors for renal dysfunction in men with obstructive and nonobstructive voiding dysfunction. J Urol 1997; 158(1):181-5. doi: 10.1097/00005392-1997 07000-00059.

5. Shin YS, Zhang LT, You JH, Choi IS, Zhao C, Park JK. Efficacy and safety of tamsulosin hydrochloride $0.2 \mathrm{mg}$ and combination of tamsulosin hydrochloride $0.2 \mathrm{mg}$ plus solifenacin succinate $5 \mathrm{mg}$ after transurethral resection of the prostate: A prospective, randomized controlled trial. Clin Interv Aging 2016; 11:1301-7. doi: 10.2147/CIA. S115042.

6. Sipal $\mathrm{T}, \mathrm{Akdere} \mathrm{H}$. The relation between the storage symptoms before and after transurethral resection of the prostate, analysis of the risk factors and the prevention of the symptoms with solifenacin. Int Braz J Urol Off J Braz Soc Urol 2020; 46(4):575-84. doi: 10.1590/S1677-5538.IBJU. 2019.0227 